\title{
Language Practices of Trilingual Undergraduate Students: Engaging One Task in Three Languages
}

\author{
Evelyn Njurai
}

Kiriri Women's University of Science and Technology, School of Science, Kasarani Campus, Kenya

\begin{abstract}
This paper explores how and why some trilingual undergraduate students of mathematics use their languages to make sense of mathematics. The paper draws from a wider study focusing on language practices of undergraduate students of mathematics in Kenya. The notion of Discourse analysis is used as an analytic tool to describe and explain the language practices of two students. In a country like Kenya where majority students acquire trilingualism through schooling, a practice is advanced where home languages are dominant languages of thinking and understanding tasks, the national language supports communication; while English, the language of learning and teaching, is a language of external communication. Further research on language practices in trilingual context is recommended.
\end{abstract}

Keywords: Trilingual, undergraduates, home language, language practices, Discourse analysis

\section{Introduction}

This paper explores how and why some trilingual undergraduate students use their languages to make sense of mathematics tasks. The concern with the language practices of trilingual students is in keeping with the need for research related to the use of languages other than the Language of Learning and Teaching (LoLT), as suggested during a symposium on "Interactions between Linguistics and Mathematical Education" held in Nairobi in 1974 [1]. The symposium [1] report noted that when mathematical ideas are presented to multilingual students in an additional language, the students may relate these ideas to how they address them in their home languages. Since the symposium, research in mathematics education particularly in Africa has focused on the use of two languages among multilingual learners. Further research among multilingual students can partly be addressed through research into the language practices of trilingual students, by viewing trilingualism as a special case of multilingualism. In fact, research [2], shows that language practices of trilingual students in mathematics education have not as yet been explored.

The argument I set forth is that trilingual undergraduate students of mathematics who are competent in LoLT switch to their home languages and the national language as resources in making sense of mathematics tasks. I draw on the schooling context in Kenya to consider how and why trilingual students use their three languages. Relevant extracts from interview transcripts are used.

\section{Who is Trilingual?}

Research into trilingualism is limited and no one definition has so far been adopted [3]. However, researchers in this field have identified quantitative and qualitative aspects that are characteristic of trilingualism which they have used to explain it [3]. The quantitative aspect of trilingualism shows that trilingualism occurs when a speaker knows three languages. The qualitative aspects are for instance, the existence of different groups of trilingual speakers, depending on both the circumstances and the social context under which they acquire and use the three languages. Further, a trilingual speaker uniquely uses his/her three languages in ways that are determined by his/her communication needs. He/she has the ability to function like a monolingual, a bilingual or a trilingual, depending on the topic, place or interlocutor. To function like a bilingual or trilingual, the speaker requires to code switch between languages. The quantitative and qualitative aspects of trilingualism suggest that while a trilingual person may share some characteristics with a bilingual and multilingual person, a trilingual is not an extension of a bilingual person but a special case of a multilingual person with specific characteristics.

\section{Situating Trilingualism in Kenya's Context}

The majority of students in Kenya acquire trilingualism at school through the practice of the Language in Education Policy (LiEP). The policy states that during the first three years of their schooling, students are taught through the medium of the home language that is predominant in the school environment. The students may represent a variety of home languages; hence the mathematics classrooms are multilingual. During this period, they are introduced to learning their home languages as well as English and Kiswahili as subjects. Neither Kiswahili nor English are first or home languages for the majority of students. English is the official language while Kiswahili is the national language and co-official language. From the fourth year of primary school, the LoLT is English; Kiswahili continues to be taught as a subject while the home languages are dropped from the academic process.

Research conducted in Kenya has explored the use of two languages, for instance Kiswahili and English or Dholuo and English. This has been particularly from a teaching perspective [4], [5]. What these studies do not tell us is how majority students in Kenya who are exposed to three languages deal with trilingualism. Thus the purpose of the research reported in this paper is to explore how and why trilingual undergraduate students use their languages to make

Volume 6 Issue 1, January 2017 


\section{International Journal of Science and Research (IJSR) \\ ISSN (Online): 2319-7064 \\ Index Copernicus Value (2015): 78.96 | Impact Factor (2015): 6.391}

sense of mathematics. The three languages under focus are: the students' home languages, the national language, Kiswahili, and the LoLT, English. Specifically, the paper responds to the questions:

1) What are the language practices of trilingual students when engaging with mathematics tasks?

2)Why do they use languages as they do?

These questions focused on the students' language practices through their verbal, written expressions and reflections on their linguistic train of thought, while they engaged with a mathematics task.

\section{Language and Discourse Analysis}

The perspective taken in this paper draws widely from Vygotsky's theory that development occurs in and through socially mediated activity and that language plays a key role in this mediation [6]. According to Vygotsky's theory, language serves first a regulative, communicative function and later becomes a tool for thinking. Language expresses thoughts through verbal and non-verbalised communication, that is, in spoken and written language, and gestures; while other thoughts remain unexpressed outside the human person [6]. It mediates communication within and between humans. Language therefore has the power to transform the way people learn, think and understand.

Research works [7] on language use show that the primary function of language is to support performance of social activities and identities and, human affiliation within cultures, social groups and institutions. When we use language, we enact certain Discourses (with capital D) in the same or different contexts, which are language plus non-language "stuff". Thus Discourse is: ways of combining and integrating language, actions, interactions, ways of thinking, believing, valuing, and using various symbols, tools, and objects to enact a particular sort of socially recognizable identity (pp. 21).

In other words, we use spoken or written language in tandem with non-language "stuff" to perform actions in the world. The actions project us as certain kinds of persons engaged in certain kinds of activities and hence we get associated with groups whose members act as we do [7]. The meanings derived in any one Discourse situation are multiple, varied and situated in context of use. Central to Discourse analysis is recognition; if the activity and identity are recognised, then one will have "pulled off" a Discourse of a sort [7, pp 23]. Discourse analysis was important for analysis of language practices of the trilingual students. The particular Discourses that apply in this paper are mathematics Discourses.

Using a situated and socio-cultural perspective and the notion of Discourses, mathematical Discourses constitute ways of combining and integrating language with other non-language "stuff", and ways of saying, doing and being in mathematics [8]. The perspective looks at the situational resources students use and ways that mathematics Discourses are relevant to the situation. It helps us to consider the non- language resources that the students are exposed to and can use when they engage with mathematics rather than focus on mathematical vocabulary. Non-language resources include the use of languages in a students' repertoire that are not necessarily the LoLT. Thus mathematical Discourses allow us to more fully describe the variety of resources that students use to communicate mathematically.

The Discourse analysis discussed can be used to analyse language practices within a one language or a multiple language environment. In this paper, the different Discourses involve use of multiple languages in one mathematical task. The language practices of some trilingual undergraduate students were analysed in an effort to understand how and why they used language as they did when they engaged with a mathematics task and the socially situated identities and activities that students enacted.

It is however important to note that Discourses are mediated through natural languages, for example English and Kiswahili. With that regard, and in the absence of literature on language practices involving trilingual students of mathematics, a discussion on language practices involving bilingual and multilingual students' sheds light on possible language practices among trilingual students.

\section{Language Practices of Bilingual and Multilingual Mathematics Students}

Harnessing students' home languages as important resources for learning has been argued as a means to improve multilingual students' participation and performance in mathematics [9], [10]. This is especially appropriate when they have limited proficiency in the LoLT, which may prevent them from expressing their mathematics ideas clearly. However, research shows that bilingual students who are competent in the LoLT also draw on their home languages to make sense of mathematics because they face interpretation challenges in mathematics [11]. Therefore, a common language practice among bi/multilingual students is that of code switching between LoLT and home languages.

\section{Code switching}

Code switching is the alternative use of two or more languages in an utterance or conversation in a more or less deliberate way [12], [13]. The alternation can involve a word, a phrase, a segment of a sentence, a sentence or several sentences. It is a common characteristic of bi/multilingual speech. While the works [12], [13] portray code switching as a verbal strategy, a corresponding non-verbal strategy of language switching has been proposed and used in research on mathematics education. Language switching refers to the use of two or more languages during solitary and/or mental arithmetic computation [14]. This involves switching between two languages when thinking through computations, portraying an internal function. While acknowledging this differentiation, in this paper, I choose to refer to all situations where students switch between languages in verbal conversations and/or in mental computations as code switching. 


\section{International Journal of Science and Research (IJSR) \\ ISSN (Online): 2319-7064}

Index Copernicus Value (2015): 78.96 | Impact Factor (2015): 6.391

Linguists explain that code switching happens for a range of purposes. They include, translating content from one language to another and to easily and efficiently express oneself [12], [13], [15]. Reasons for which translation has been used in mathematics classrooms include familiarity with the other language(s) and hence the speaker may habitually translate some words [16]. Translation also happens as a constant practice with the ultimate goal of transforming information into a more usable representation [17]. For instance, Vietnamese mathematics students translated problems from English into Vietnamese while reading and thinking them through [11]. They later translated them back into English to make the ideas compatible with the classroom language situation. Some bilingual mathematics students also switch between languages in order to express themselves easily and efficiently [18]. The switching is not necessarily the result of the students not knowing a word or a phrase in one language; rather, they are taken to facilitate the use of words or phrases in the other language.

The discussion above shows that exploring students' mathematical participation and performance by focusing on code switching between the LoLT and home languages, broadens the view of understanding bi/multilingual students' mathematical ability. However, this view remains narrow since multilingual students have other languages in their repertoire [19]. In fact, trilingual students' participation in mathematics Discourse in their third language, which is their national language, has not yet been explored.

\section{Method}

The wider study, from which this paper draws, was qualitative and involved 15 trilingual undergraduate engineering students taking mathematics in their programs at one public university located in the central part of Kenya [20]. Research ethics were taken into account prior to the research. The university draws its students' population from all over the country and hence the students have varied language backgrounds. Data was collected using questionnaires, clinical and reflective interviews. All the students were academically proficient in the LoLT and in mathematics. Given the opportunity to choose the language for the interviews, all the participants opted to be interviewed in English. The findings presented in this paper are from one of the themes that emerged from two students who engaged in the mathematics task in three languages.

The two students S13 and S14 were in their first year of study taking their degree in Civil Engineering. Their home languages were Kikuyu and Dholuo respectively. Baseline information showed that S13 spoke Kikuyu more than he did Kiswahili or English at home with family members. All three languages were important for his individual engagement with mathematics and he switched between English and Kiswahili when discussing mathematics with his peers and lecturers. S14 commonly used Dholuo with his family; English and Kiswahili were rare languages of communication. When doing mathematics on his own and with his peers, he commonly switched between English and Kiswahili, and he communicated with his lecturers in English.
The task that the students engaged in was;

A hall can accommodate 600 chairs arranged in rows. Each row has the same number of chairs. The chairs are rearranged such that the number of rows is increased by 5 but the number of chairs per row is decreased by 6 .

(a) Find the original number of rows of chairs in the hall.

(b) After the rearrangement, 450 people were seated in the hall leaving the same number of empty chairs in each row. Calculate the number of empty chairs per row.

To guide the analysis, the following questions written in English were asked directly or indirectly of each student: Which other languages did you use as you engaged with the task? Which other languages do you use while engaging with mathematics either in discussion groups or alone? Why did you use the language(s) as you did? All code switching instances were noted.

\section{Findings and Discussion}

A recurrent theme in the analysis was that the students switched between English, their home languages and Kiswahili either internally or externally. The home languages were the dominant languages that the students used to think and understand the task and hence make sense of it. They translated part or the whole task to home languages because they were more familiar with the languages and for S13 because code switching was a constant language practice. Furthermore, switching to Kiswahili facilitated ease in their communication.

\subsection{Thinking Through and Understanding the Task}

S13 explained how and why he used his home language, Kikuyu. It was necessary for his thinking and understanding of the task.

S13: For one, when you, the first part when I was reading it, when I was silent, I was trying to get is "what is the question trying to ask?" and I could visualise it in my own language, because / this language is not so...is not "haikuangi ati common kwa kila mtu" [it (English) is not common to everyone]. So at a certain point I could read the question if I have not understood then I try to figure out, "what does it mean?" If I'm given about this information, now I have to digest this information bit by bit in my own language. Then / from there after I have understood, after I have understood now the question, I could now be able in a position to write or answer the question.

S13 read the task in English, but thought through and imagined what was expected of him in his own language, particularly the parts he thought he did not understand. He switched to his own language because English was not his everyday language. After having understood what the task required of him he proceeded to answer it. His own language was his home language, Kikuyu:

$\mathrm{R}$ :You mentioned that you usually visualise it in your own language; what is this own language? 


\section{International Journal of Science and Research (IJSR) \\ ISSN (Online): 2319-7064}

Index Copernicus Value (2015): 78.96 Impact Factor (2015): 6.391

S13: Most of the time ... after I read a certain piece of question, I have to think it in my language now, my first language...now after thinking in Kikuyu then I could now go to the question, now I have understood in a way that I can now react to the question what it is asking.

\section{$\mathrm{R}:$ Does this happen always?}

S13: Most of the time; if for example you can give me a question I lack to understand in a way that I can speak it in my own language, most of the time I will not be able to answer that question properly.

He used Kikuyu in the interpretation of the task. The use of Kikuyu was not unique to this task; it was a common practice for S13. He revealed that if he did not understand a question in Kikuyu, he would most likely not answer it correctly. This shows that while he read the tasks in English, he used Kikuyu to think and to seek understanding of the task. He explained how he used Kikuyu in this particular task:

S13: For example, in this part, I could read this one \{referring to the first sentence in the question\} I know what is talking about in my own language. Then after the first sentence then I continue \{with all other sentences\} then I can get the real picture of what the question is talking about. For example, here \{reads the first sentence\}. Now I could ask I could visualise in my mother tongue, "this is 600 / how could they be arranged? Yes, they are in a room but how could they be arranged?" After I think it in my own ways now I come back to the questions after I have understood what is in me...

$\mathrm{R}$ : Is it something you can write?

S13: I write it in my own language? $\{\mathrm{R}$ nods and $\mathrm{S} 13$ goes on to write the information part of the question in Kikuyu and then reads it aloud\}.

Nyumba iganagira itĩ magana matandatu ibangitwo na mĩhari. Omũhari ukoragwa na itĩ ciganaine. Itĩ ni ciabangurirwo na ikibangwo ringĩ, na itĩ iria ciari muhari umwe ikĩnyiha na ithatatu na mihari ikiongerereka na itano. [A house/building can accommodate 600 chairs arranged in rows. Every row has an equal number of chairs. The chairs were rearranged, and the chairs in each row reduced by six and the rows increased by five].

This is how now I understood the question in my own language. Now this is how I understood it in this format, after I understood it in this format, I could now translate this information in now in English, in my own way.

Although S13 had earlier indicated that he used Kikuyu when faced with challenges to his understanding, it seems he sought understanding of the whole task by translating it, sentence by sentence, into Kikuyu. He achieved the desired understanding of the task in Kikuyu then translated what he was writing into English. Up to this point, this trilingual student demonstrated how and why he switched between English and his home language.
For S14, the use of his home language, Dholuo, was as a result of challenges of reinterpreting part of the task in English.

S14: I involved it $\{$ Dholuo $\}$ at the stages where I was not able to interpret in terms of English.

\section{R:Like which parts?...}

S14: In part (b) I had to involve, I was a bit confused in terms of these people $\{450\}$ and the number of seats here. I had to involve Dholuo and Kiswahili so that I interpret that each chair was supposed to accommodate an individual. So depending on the equation that I got in part (b), I had to equate to the number of people so that I could solve it.

\section{R: So did you translate the whole of part (b)?}

S14: In mother tongue, yeah.

$\mathrm{R}$ :How did it go like? If you can write $\{\mathrm{S} 14$ writes the translation of part (b) in Dholuo .

S14: "Ka ji 450 obedo to gi wuoyo kombe, kombe ma odong' onego bed ni ting'o ji 150". [If 450 people are seated and they rearrange the chairs, the remaining chairs should accommodate 150 people] \{He then read out the translation\}. I set out the equation for the remaining chairs... \{Inaudible\}.

$\mathrm{R}:$ And that assisted in getting the solution?

S14: Yes, because in stage (a) I was now having the equation, how I could put it so that I solve it for the number of empty chairs that was where there was a problem.

S14 had some initial difficulties with (a); he reworked the task to completion and arrived at the expected solution. But it seemed the challenges posed in (a) did not require him to switch languages as he did in (b), where he had difficulties in the interpretation of the task and this caused some confusion. He needed to link the solution arrived at in (a) to the requirements of (b). In order to do so, S14 translated part of (b) into Dholuo. The translation assisted him in linking (a) to (b) and arriving at the solution to (b). From S14's account, it is clear that Dholuo was used as a linguistic resource when he faced interpretation challenges in English. It emerged as the dominant language that shaped his understanding when he was faced with interpretation challenges.

\subsection{Ease of Communication}

The students seemed to switch to a third language, Kiswahili, for various reasons. In his verbal explanation during the two interviews, S13 switched to Kiswahili, saying "kwa hivyo, [so that]" "ukuje useme [you say]" and "hapa [here]". The meaning of the words or phrases does not reflect any translation of words in the task and their use did not suggest that S13 derived mathematical meaning from them. Rather, 


\section{International Journal of Science and Research (IJSR) \\ ISSN (Online): 2319-7064}

Index Copernicus Value (2015): 78.96 Impact Factor (2015): 6.391

the words seemed to have been taken to facilitate the use of words or phrases in the language. In my view, S13 used the words in order to express himself easily like bilingual students do [11], [18]. In fact, he had earlier on indicated that English is not "common" to him and it could be that he used the next official language as means to communicate with the researcher. While this use of Kiswahili was explicit, S13 explained that he also used it to think. This only happened after he gained understanding in his home language.

I explored how and why he did so:

R:At certain times as you went along you used other languages.

\section{S13: Yeah, mostly I usually use Kiswahili.}

R:How does it feature in your work, as you do it? .... What role does it $\{$ Kiswahili\} play in responding to the task\}?

S13: This one maybe after understanding it in this way in reference to the Kikuyu translation\}, because Kiswahili is the language which is closer to my language, now I come to use it severally...If it's come on mathematics or maybe sciences, I will have to think in those two languages, I read in this one \{English\}, then in the process I will come now to use this Kiswahili in the meanwhile.

S13 initially seems to be hedgy on his use of Kiswahili but subsequent utterances make it clear that it is not only in mathematics that he switches to Kiswahili. He claims to use Kiswahili in mathematics only after he has gained understanding in Kikuyu, because Kiswahili is close to Kikuyu. It is not clear whether this occurred in a mixture of Kiswahili and Kikuyu or whether there was a distinction between their functions prior to translating back to English for reporting purposes. However, it is clear that he switches first to Kikuyu to think and understand the task and then if need be to Kiswahili. The closeness suggests that while he was most familiar with Kikuyu, when it came to Kiswahili and English languages, he was more familiar with Kiswahili. This may explain why he used Kiswahili only after gaining understanding in Kikuyu, and finally switched to English so as to report.

S14 explained his interpretation of the task in the LoLT. He however switched from English to Kiswahili to emphasise the claim that the task required the formation of a quadratic equation. The use of Kiswahili was not related to the mathematics but rather to the ease of using Kiswahili words to emphasize his claim. While this switch to Kiswahili was the only notable example of code switching practice in the interpretation, during the reflective interview S14 revealed that he switched to Kiswahili almost throughout the engagement with the task to read some numerals. Prior to the utterances in extract below, S14 explained his use of Dholuo and then continued to explain how and why he used Kiswahili.

R:There was also the mention of Kiswahili, tell me how you used it in this question.

S14: Kiswahili I used it almost throughout. I used it when I was referring to the numbers here like 600 , like 6 , like 5 .

R: Why do you use Kiswahili for the numerals?

S14: I always tend to read them \{in Kiswahili\}.

$\mathrm{R}$ :So even when you are reading this \{quadratic\} formula here, that $4 \ldots$ \{Referring to the constant value 4 in the quadratic formula\}

$$
x=\frac{-b \pm \sqrt{b^{2}-4 a c}}{2 a}
$$

S14: But not such cases only at times ndio natumia [it's what I use], and I actually think maybe because I'm living with people... \{Inaudible $\}$... so most of the time we speak Kiswahili.

S14 read some numbers in Kiswahili, such as the numbers of chairs, rows and people. These were numbers of countable items and not numbers in equations. While initially he could not explain the language practice, later S14 said he thought he did so because Kiswahili was the dominant language for social communication in his residential area. His environment had socialized him to visualize such numerical figures in the national language. Therefore, S14 habitually read such numbers in Kiswahili even when the task was presented in English as was the case in the task that was at hand. It seems it was easier and more efficient to think of the numbers in Kiswahili as was the habit. Habitual reading of words in one language and not in the given language is not new in mathematics education. In fact, Persian-English bilinguals switched to read English words, for instance "girl" was read as "doktar" in Persian because of the habitual use of this word in Persian [16]. Therefore, S14 switched to Kiswahili to emphasise his claim and due to the habitual use of the national language and subsequently ease in communication.

\section{Emerging Discourses}

At the beginning of this paper, I set forth the argument that trilingual undergraduate students who are competent in LoLT use their home languages and the national language as resources to make sense of a mathematics tasks. The analysis has shown that the Discourses that have emerged are that the two trilingual undergraduate students identified with the use of three languages in their mathematics engagement. The languages used in verbal communication, writing and thinking in relation to their social culturally defined experiences, shaped the identities they enacted and the activities they were engaged in when working on the task. They used their three languages according to their communication needs.

S13 read and wrote the task in English, interpreted it in Kikuyu, and then perhaps used Kiswahili before translating back into English for reporting purposes. S13 not only switched and translated the task content into either of these two languages; rather this was a common practice in other tasks. The use of Kiswahili in his verbal communication is 


\section{International Journal of Science and Research (IJSR) \\ ISSN (Online): 2319-7064}

Index Copernicus Value (2015): 78.96 | Impact Factor (2015): 6.391

associated with the ease of communicating his understanding of the task. These findings reflect S13's baseline information that all three languages were important for his individual engagement with mathematics. S14 mainly used English in his verbal explanation and in all his written work. He asserted his claims in Kiswahili; furthermore, he had developed a habit of reading numbers of countable items in Kiswahili. S14 switching to Kiswahili could be seen as a result of his familiarity with certain words and numbers in the Kiswahili language, and not because of a lack of understanding of such words [8]. When he needed to reinterpret part of the task; he did so by switching and translating part of the task content to Dholuo in order to form the required understanding. In fact, these findings agree with the baseline information provided by S14, that when engaging with mathematics he switches between English and Kiswahili. The switch to Dholuo was a reaction when $\mathrm{S} 14$ faced interpretation challenges. In the cases of both S13 and S14 switching to home language was an internal function, while switching to Kiswahili was both an internal and external function.

From the discussion above, one of the purposes of code switching was to think through and understand the task. This was done by switching between English and their home languages. They translated whole sentences or the entire task into their respective home languages. This switching was aimed at interpreting the task. The students' code switching practice to their home languages provided access to mathematics and supported the finding of [10] that home languages are resources that aid students' understanding of mathematics.

The other purpose of code switching was ease of communicating their understanding but not necessarily to derive the meanings of any particular words. This was particularly seen when switching to Kiswahili. S14 habitually switched to read numbers while S13 switched after having understood the task in Kikuyu.

From the analysis, it is clear that all the languages were not used equally; home languages were dominant languages for conceptual engagement, Kiswahili was used to ease communication and English was positioned as a dominant language for communicating with the researcher during the interviews. The home languages were positioned as languages of thinking and understanding the task, an internal function; while Kiswahili and English were for both thinking and verbal communication, external and internal functions. It is clear that code switching between the languages provided an opportunity for the languages to work together to make mathematics accessible to the students. The findings support the claim that trilingual students can use all three languages in their repertoire [3] and that students who are competent in LoLT also switch to their home languages to engage with mathematics [11].

\section{Conclusion}

The findings presented in this paper contribute to the global debate on language practices among students who are users of more than one language. The study partly attends to the research gap in trilingual context. The findings are particularly relevant to the Kenyan multilingual context, where majority of students are exposed to three languages at school, and other similar contexts like Catalan, India and Malawi. Research in mathematics education in the context of trilingualism is recommended to inform how students use their three languages and hence to support the findings of this study.

\section{Acknowledgement}

I would like to acknowledge the valuable contribution and support of my supervisor Prof Mamokgethi Phakeng, which she provided during the production of the larger study. The study was made possible with the funding and support from the University of South Africa.

\section{References}

[1] UNESCO. The interactions between linguists and mathematics education. UNESCO, CEDO \& ICMI 1974.

[2] Phakeng, M. S, Mathematics education and language diversity: past, present and future. In Proceedings of Fourth Africa Regional Congress of International Commission on Mathematics Instruction, pp. 14-24, Maseru, Lesotho, 2013.

[3] Hoffmann, C, Towards a description of trilingual competence. International Journal of Bilingualism, 5(1), pp. 1-17, 2001

[4] Bunyi, G, Multilingualism and discourses in primary school mathematics in Kenya. Language, Culture and Curriculum, 5(4), pp. 492-504. 1997.

[5] Cleghorn, A., Merrit, M., \& Abagi, J, Language policy and science instruction in Kenya primary schools. Comparative Education Review, 23(1), pp. 21-39, 1989.

[6] Vygotsky, L, Thougtht and Language. Cambridge: MIT Press, 1986.

[7] Gee, J, An introduction to Discourse analysis: Theory and Method (Second Ed). London: Routledge 2005.

[8] Moschkovich, J, A situated and sociolcultural perspective on bilingual mathematics learners. Mathematical Thinking and Learning, 4, pp. 11-19, 2002.

[9] Setati, M., \& Adler, J, Between languages and discourses: language practices in primary multilingual mathematics classrooms in South Africa. Educational Studies in Mathematics, 43, pp. 243-269, 2000.

[10] Setati, M., Molefe, T., \& Langa, M, Using language as a transparent resource in teaching and learning mathematics in a Grade 11 multilingual classroom. Pythagoras, 67, pp. 14-25, 2008.

[11] Clarkson, P, Australian Vietnamese students learning mathematics: high ability bilinguals and their use of their languages. Educational Studies in Mathematics, 64, pp. 191-215, 2006.

[12] Baker, C, Foundations of bilingual education and bilingualism. Clevedon, Avon: Multilingual Matters, 1993. 
[13] Grosjean, F, Life with two languages. Cambridge, MA: Havard University Press, 1992.

[14] Moschkovich, J, Using two languages when learning mathematics. Educational Studies in Mathematics, 64, pp. 121-144, 2005.

[15] Cohen, A, In which language do/should multilinguals think? Language, Culture and Curriculum, 8(2), pp. 99-113, 1995.

[16] Parvanehnezhad, Z., \& Clarkson, P, Iranian bilingual students' reported use of language switching when doing mathematics. Mathematics Education Research Journal, 20(1), pp. 51-81, 2008.

[17] Kern, R. G, The role of mental translations in second language reading. SSLA, 16, 441-461, 1994.

[18] Planas, N. (2011). Language identities in mathematics writing about group work in their mathematics classroom. Language and Education, 25(2), 129-146.

[19] Gorgorio, N., \& Plana, N, Teaching mathematics in multilingual classrooms. Educational Studies in Mathematics, 47, pp. 7-33, 2001.

[20] Njurai, E. W., Language practices of trilingual students engaging with mathematics in Kenya, Unpublished Phd thesis, University of South Africa, Pretoria, 2015.

\section{Author Profile}

Evelyn Njurai is a senior lecturer and coordinator of mathematics programs in the School of Science at Kiriri Women's University of Science and Technology. Her research interests include mathematics education in context of language diversity and using technology to teach and learn mathematics. 